\title{
Translated Poisson Approximation for Markov Chains
}

\section{A.D. Barbour · Torgny Lindvall}

Published online: 15 October 2008

(C) Springer Science+Business Media, LLC 2008

\section{Erratum to: J Theor Probab (2006) 19: 609-630 DOI 10.1007/s10959-006-0047-9}

The geometrically small part of the error term in Lemma 4.1 was incorrectly treated in (4.7), and, as a result, the definition of the quantity $b_{i}$ in the lemma needs to be altered to:

$$
\begin{aligned}
b_{i}:= & \tilde{\varphi}(n)\left\{\frac{1}{2} \mathbb{E}\left\{\left|Y_{i}\left(A_{i}-A_{i}^{\prime}\right)\right|\left(\left|A_{i}\right|+\left|A_{i}^{\prime}\right|\right)\right\}\right. \\
& +\mathbb{E}\left\{\left|Y_{i}\left(A_{i}-A_{i}^{\prime}\right)\right|\left(H\left(T_{i}^{+}-i\right)+H\left(i-T_{i}^{-}\right)\right)\right\} \\
& \left.+\mathbb{E}\left\{\left|Y_{i}\left(A_{i}-A_{i}^{\prime}\right)\right|\right\}\left\{\mathbb{E}\left|A_{i}\right|+\mathbb{E}\left(H\left(T_{i}^{+}-i\right)+H\left(i-T_{i}^{-}\right)\right)\right\}\right\} \\
& +\gamma_{i},
\end{aligned}
$$

where, for $1 \leq i \leq n / 2$,

$$
\begin{array}{ll}
\gamma_{i}=2 \mathbb{E}\left\{\left|Y_{i}\left(A_{i}-A_{i}^{\prime}\right)\right|\left(I\left[T_{i}^{+}-i>n / 4\right]+\mathbb{P}\left[T_{i}^{+}-i>n / 4\right]\right)\right\}, & 1 \leq i \leq n / 2 \\
\gamma_{i}=2 \mathbb{E}\left\{\left|Y_{i}\left(A_{i}-A_{i}^{\prime}\right)\right|\left(I\left[i-T_{i}^{-}>n / 4\right]+\mathbb{P}\left[i-T_{i}^{-}>n / 4\right]\right)\right\}, & n / 2<i \leq n .
\end{array}
$$

The online version of the original article can be found under doi:10.1007/s10959-006-0047-9.

A.D. Barbour $(\bowtie)$

Angewandte Mathematik, Winterthurerstrasse 190, 8057 Zürich, Switzerland e-mail: a.d.barbour@math.uzh.ch

T. Lindvall

School of Mathematical Sciences, Chalmers and GU, 41296 Göteborg, Sweden e-mail: lindvall@math.chalmers.se 
The quantity $\tilde{\varphi}(n)$ is smaller than the quantity $\varphi(n)$ appearing in the original statement of the lemma, and hence is still of the desired order $O\left(n^{-1 / 2}\right)$ under assumption (3.7). The term $\gamma_{i}$ is typically very much smaller.

Changing Lemma 4.1 leads to a minor change in the specification of the bound in Theorem 4.2, again without altering the main contribution. The remainder of the paper is unchanged. A corrected version, giving the proof of the new version of Lemma 4.1, is to be found at

$$
\text { arXiv:0810.0599v1 [math.PR] }
$$

